\title{
The Veterans Health Administration's Contribution to Cancer Education
}

\author{
Maria Curcio Bishop ${ }^{1}$
}

Published online: 2 January 2020

(C) American Association for Cancer Education 2020

Quality outcomes of cancer education are increasingly the result of academic, community, and government collaborations. The Veterans Health Administration (VHA) has been a significant contributor to this effort. The VHA is the USA's largest integrated healthcare system. This system provides care at 1255 facilities, including 170 medical centers and 1074 outpatient sites, serving 9 million enrolled veterans each year [1].

The VHA offers several educational programs for veterans with cancer and their families, ranging from enhancing knowledge about prevention and treatment to end-of-life care. These programs also provide a rich learning environment for healthcare providers in training, and many VHA hospitals are major teaching partners of affiliated universities. The programs offered promote shared decision-making, an interdisciplinary approach to providing care, and respect for culture.

My colleagues and I have had the pleasure of working with several of the VHA programs. Our patients have benefitted from many available programs, including tobacco cessation, nutrition, mindfulness, and caregiver support. The VHA also has a strong commitment to ethics and research, both of which support education. To provide a greater understanding of VHA resources, I would like to share three programs in more detail that highlight the VHA contribution to cancer education.

The VHA pharmacy program recognizes that pharmacists are essential to optimizing the care of patients with cancer. Their expertise in medication use promotes wellness, prevents and manages diseases, ensures patient safety, and optimizes health outcomes in collaboration with the interdisciplinary oncology team. The goal of the pharmacy program is to provide veterans with evidence-based medication information to

Maria Curcio Bishop

mbishop@email.arizona.edu

1 Division of Hematology and Oncology, University of Arizona Cancer Center, PO Box 245024, 1515 N Campbell Ave, Tucson, AZ 85724-5024, USA enable them to work with their healthcare team in making informed decisions about their preferences for care.

The US Department of Veterans Affairs (VA) Pharmacy Residency Program is the largest program for advanced clinical pharmacy training in the country, with several postgraduate Year 1 (PGY-1) and Year 2 (PGY-2) programs. The program is responsible for the education of approximately 607 residents annually in 236 American Society of Health-System Pharmacists (ASHP)-accredited residencies [2]. There are five PGY-2 Oncology Pharmacy Residency Programs in the USA dedicated to training clinical oncology specialists. Research and academic opportunities are mentored by experienced oncology pharmacists. Oncology pharmacists and pharmacy residents are active members of the interdisciplinary oncology treatment team. They provide education for hematology/oncology fellows, internal medicine house staff, medical students, nurses, and patients and their families. Chemotherapy and immunotherapy, symptom and pain management, and treatment toxicity are just a few examples of the topics they address. With the increasing complexity of treatment options and their associated toxicities, the contributions of pharmacists are essential to providing standard-of-care therapies appropriately and safely while enhancing our patients' quality of life.

The VHA is committed to providing compassionate care to veterans at the end of life. Hospice (comfort) care is provided to patients and their families if the patient has a terminal illness and is expected to live 6 months or less, and is no longer seeking treatment other than palliative care. Hospice care can be provided in a variety of settings, such as the veteran's home, an assisted-care facility, or an inpatient unit. The VHA collaborates with community and home hospice agencies to provide care in outpatient settings. Since hospice care is part of the VHA Standard Medical Benefits Package, all enrolled veterans are eligible if they meet the clinical need for the service.

Both inpatient and outpatient hospice care programs provide services that relieve suffering and help control symptoms in a way that respects personal, cultural, and religious 
beliefs and practices. Hospice care also provides bereavement support to the patient's family. Patients and their families meet with an interdisciplinary care team to develop a plan of care together that meets their medical, social, spiritual, and psychological needs. Hospice providers teach patients and caregivers about symptom management and physiological changes that occur during the dying process. The hospice and palliative care team also sponsor an elective for internal medicine residents and hematology/oncology fellows. Additionally, they are a major teaching partner of university-based palliative care fellowships.

Historically, Native American and Alaska Natives have had the highest record of service per capita compared with other ethnic groups, with over 140,000 veterans selfidentifying with one of these groups [3]. The US Department of Defense estimates that there are currently over 14,000 active-duty Native service members in the US Armed Forces [4]. The VHA has Native American and Alaskan Native healthcare liaisons available to educate care providers about the culture and care of our Native American veterans. The Traditional Way Culture Sensitivity Workshop and The Gathering of Healers are programs that provide a forum for this education. The liaisons also play a significant role in educating patients and their families. Many veterans live far distances from their healthcare providers. The liaisons make home visits and are available to take students and residents as part of their outpatient clinical training.

For additional information about these and other educational programs offered by the VHA Administration, please refer to their website: https://www.va.gov/health.

Our next cancer education conference will be the European Association for Cancer Education (EACE) 33rd Annual Scientific Meeting in Krakow, Poland, on April 1-3, 2020. The program theme has been designed to cover a range of topics within the context of sharing knowledge and sharing practices. Strands within the program include patient-centered communication, shared decision-making, cultural diversity, politics and policy, risk assessment and management, and education for patients, their families, and the community. Travel grants are available to facilitate your attendance. We encourage you to submit abstracts for oral papers and/or posters, the deadline for which is March 22, 2020.
A new feature of the annual EACE meeting is a preconference course in essential skills in cancer education. The title of the course is "Leadership: Leading and Influencing Change in Cancer Education." This 1-day international education forum, hosted by EACE and AACE, will include presentations by leading international educators, interactive sessions, breakout sessions, small-group discussions, and networking opportunities with mentors. The event will bring together international cancer educators across disciplines to exchange ideas and discuss topics such as leadership in cancer education and how to lead and influence change in cancer education at different organizational or personal levels. This course is aimed at practicing physicians, other healthcare providers, basic scientists, clinicians, and doctoral students who would like to learn leadership styles in cancer education. Attendees will receive educational credit for this course.

In closing, I would like to personally thank all our reviewers for the time they volunteer to review manuscripts, share their expertise, and mentor junior reviewers and authors. If you would like to become a reviewer, please contact me. If you do not have experience reviewing manuscripts but would like to learn under the guidance of an experienced reviewer, I would be pleased to introduce you to a mentor.

Best wishes for a happy and healthy New Year!

\section{References}

1. US Department of Veterans Affairs. Veterans Health Administration: Providing Health Care for Veterans. https:/www.va.gov/health. Accessed 9 December 2019

2. US Department of Veterans Affairs. Pharmacy Benefits Management Services: VA Pharmacy Residency Program, National Program Brochure. https://www.pbm.va.gov/PBM/education/residency/ generalinfo/2016_2017_National_VA_Residency_Brochure.pdf. Accessed 9 December 2019

3. US Census Bureau. American FactFinder. https://factfinder.census. gov/faces/tableservices/jsf/pages/productview.xhtml?src=bkmk\#. Accessed 9 December 2019

4. Military OneSource (2018) Demographics Profile. http://download. militaryonesource.mil/12038/MOS/Reports/2018-demographicsreport.pdf. Accessed 9 December 2019

Publisher's Note Springer Nature remains neutral with regard to jurisdictional claims in published maps and institutional affiliations. 